\title{
EXTENDING EQUIVARIANT MAPS FOR COMPACT LIE GROUP ACTIONS ${ }^{1}$
}

\author{
BY JAN W. JAWOROWSKI \\ Communicated by Morton L. Curtis, December 21, 1972
}

\begin{abstract}
Extensions of maps are studied in the category of spaces with actions of a compact Lie group $G$. If $G$ acts on a finite dimensional compact metric space $X$ with a finite number of conjugacy classes of isotropy subgroups, if $\tilde{X}$ is a closed equivariant subspace of $X$ such that the action on $X-\tilde{X}$ is free and if $f: \tilde{X} \rightarrow Y$ is an equivariant map to a compact metric space $Y$ with a $G$-action, then an equivariant neighborhood extension of $f$ exists provided that $Y$ is an ANR; if $Y$ is an AR, then $f$ can be equivariantly extended over $X$.
\end{abstract}

1. Introduction. In previous papers [2] and [3], an extension theorem for equivariant maps in the category of spaces with periodic homeomorphisms was proved. That theorem was then applied to a characterization of equivariant absolute neighborhood retracts and absolute retracts in this category. The purpose of this note is to announce results which extend some of the results of [2] and [3] from the category of $\boldsymbol{Z}_{p}$-actions to the category of compact Lie group actions. Detailed proofs will appear in a forthcoming paper.

The following theorem is the main result of this paper.

(1.1) THEOREM. Let $G$ be a compact Lie group acting on a finite dimensional compact metric space $X$ with a finite number of conjugacy classes of isotropy subgroups; and let $\tilde{X}$ be a closed equivariant subspace of $X$ containing all the fixed points of the elements of $G$ different from the identity. Let $G$ act on a compact metric space $Y$ and let $f: \widetilde{X} \rightarrow Y$ be an equivariant map. Then:

(i) If $Y$ is an $A N R$, there exists an equivariant extension $g: U \rightarrow Y$ of fover an equivariant neighborhood $U$ of $\tilde{X}$ in $X$;

(ii) If $Y$ is an $A R$, there exists an equivariant extension $g: X \rightarrow Y$ of $f$ over $X$.

As it was pointed out in [2] and [3], the problem of equivariant extension maps is not trivial even for $\boldsymbol{Z}_{2}$-actions, that is, for spaces with involutions, if they are not fixed point free. The significance of this result

AMS (MOS) subject classifications (1970). Primary 54C15, 54C20, 54C55, 55F55, 57E15; Secondary $54 \mathrm{H} 15$.

Key words and phrases. Group action, compact Lie group, equivariant map, equivariant extension, singular fibration, singular principal fibration, cross-section, absolute neighborhood retract.

${ }^{1}$ This work was done while the author held a visiting professorship at the University of Heidelberg in the summer term of 1972. 
lies, of course, in the fact that $Y$ is not assumed to be an "equivariant" ANR or AR. This theorem leads, however, to a characterization of equivariant retracts; it will be the subject of a forthcoming paper [4].

The proof of Theorem (1.1) is based on the following idea. An action of a topological group $G$ on a space $X$ may be regarded as a "principal fibration with singularities", the singularities being due to the existence of fixed points. An equivariant map $f$ determines a cross-section in the "associated singular fibration"; and the problem of extending $f$ over the free part of the action amounts to that of extending the cross-section from the singular part to the regular part of the associated fibration. A useful tool in the construction of the extension is a linearization of a compact Lie group action due to G. D. Mostow; i.e., an equivariant embedding of the space in a Euclidean space with an orthogonal $G$ action.

2. Group actions and singular fibrations. If $p: E \rightarrow B$ is a map and $\bar{B}, \widetilde{B}$ are complementary parts of $B$ then the restrictions $\bar{p}: \bar{E} \rightarrow \bar{B}$, and $\tilde{p}: \widetilde{E} \rightarrow \widetilde{B}$, where $\bar{E}=p^{-1} \bar{B}$ and $\widetilde{E}=p^{-1} \widetilde{B}$, will be called complementary parts of $p$; we shall also say that $\bar{p}$ is the part of $p$ over $\bar{B}$, and the partition of $p$ into $\bar{p}$ and $\tilde{p}$ will be denoted by $p=(\bar{p} \mid \tilde{p})$. The part $\bar{p}$ is said to be open (resp. closed) if $\bar{B}$ is open (resp. closed).

If $p=(\bar{p} \mid \tilde{p})$ is a partition of $p: E \rightarrow B$ such that $\bar{p}$ is a locally trivial (numerable) fibration then $p$ will be said to be a singular fibration with a regular part $\bar{p}$ and a singular part $\tilde{p}$.

Let Top ${ }^{G}$ be the category of left actions of a group $G$ on topological spaces. Its objects are maps $\alpha: G \times X \rightarrow X$ satisfying the usual conditions; its subobjects are also called equivariant subspaces; and the morphisms in Top ${ }^{G}$ are also called equivariant maps. If $\bar{X}$ and $\widetilde{X}$ are complementary equivariant subspaces of $X$ with an action $\alpha: G \times X \rightarrow X$, then, just as above, we speak of complementary actions $\bar{\alpha}: G \times \bar{X} \rightarrow \bar{X}$ and $\tilde{\alpha}: G \times \tilde{X} \rightarrow \tilde{X}$ defined by $\alpha$ on $\bar{X}$ and $\tilde{X}$ and we write $\alpha=(\bar{\alpha} \mid \tilde{\alpha})$. If $\tilde{\alpha}$ is free, then $\tilde{\alpha}$ is called a regular part of $\alpha$; and $\tilde{\alpha}$ is then the corresponding singular part. The corresponding identification maps to the orbit spaces will be denoted by $p=p^{\alpha}: X \rightarrow X / \alpha, \bar{p}=p^{\bar{\alpha}}: \bar{X} \rightarrow \bar{X} / \bar{\alpha}$ and $\tilde{p}=p^{\tilde{\alpha}}: \tilde{X} \rightarrow \tilde{X} / \tilde{\alpha}$. Provided that $G$ is a compact Lie group and $X$ is completely regular, $\bar{p}$ is then a principal $G$-fibration. For this reason, $p=(\bar{p} \mid \tilde{p})$ is called a singular principal $G$-fibration with a regular part $\bar{p}$ and a singular part $\tilde{p}$.

If $\alpha: G \times X \rightarrow X$ is a $G$-action and $f: B \rightarrow X / \alpha$ is a map, then $f$ induces in a natural way a $G$-action $\beta=f^{*} \alpha: G \times Z \rightarrow Z$, where $Z$ is the space of the fibration $f^{*}\left(p^{\alpha}\right)$ induced by $f$ from $p^{\alpha}$.

Let $\alpha: G \times X \rightarrow X$ and $\beta: G \times Y \rightarrow Y$ be actions of $G$ on spaces $X$ and $Y$. Then by the $G$-action on $X \times Y$ associated to $\alpha$ and $\beta$ we mean the composition 


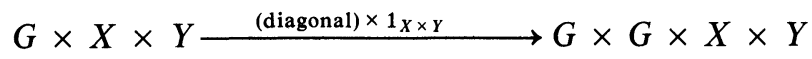

$$
\cong G \times X \times G \times Y \stackrel{\alpha \times \beta}{\longrightarrow} X \times Y \text {. }
$$

If $\gamma: G \times X \times Y \rightarrow X \times Y$ is the action associated to $\alpha$ and $\beta$ then the first projection $X \times Y \rightarrow X$ is an equivariant map $\gamma \rightarrow \alpha$ and thus induces a map

$$
q=q^{\gamma}:(X \times Y) / \gamma \rightarrow X / \alpha .
$$

The map $q$ will be called the singular fibration associated to the singular principal fibration $p^{\alpha}$ and to the action $\beta$. To a regular part $\bar{\alpha}$ of $\alpha$ there corresponds a regular part $\bar{q}$ of $q$; it is then just the fibration with fibre $Y$ associated to the principal fibration $p^{\bar{\alpha}}$, the regular part of $p$.

3. Equivariant maps and cross-sections. It was first pointed out by A. Heller [1] that an equivariant $\operatorname{map} f: X \rightarrow Y$ determines a cross-section $\varphi^{f}: X / \alpha \rightarrow(X \times Y) / \varphi$ of $q^{\gamma}$; specifically, $\varphi^{f}$ is the quotient map induced by the equivariant map $(1, f): X \rightarrow X \times Y$. We shall call $\varphi^{f}$ the crosssection associated to $f$. Conversely, Heller proved that if the principal fibration $p^{\alpha}$ is regular, then every cross-section $\varphi$ of the fibration $q^{\gamma}$ associated to $p^{\alpha}$ and $\beta$ determines an equivariant $\operatorname{map} f: X \rightarrow Y$ such that $\varphi^{f}=\varphi$. The following theorem is a relativization of Heller's result to the case of singular actions :

(3.1) THEOREM. Let $\alpha$ and $\beta$ be actions of a topological group $G$ on spaces $X$ and $Y$ as in (1.1) and let $p=p^{\alpha}: X \rightarrow X / \alpha$ be the corresponding singular principal fibration. Let $q=q^{\gamma}:(X \times Y) / \gamma \rightarrow X / \alpha$ be the singular fibration associated to $p$ and $\beta$. Suppose that the singular part $\tilde{\alpha}: G \times \tilde{X} \rightarrow \tilde{X}$ of $\alpha$ is such that $\tilde{X}$ is closed in $X$. Let $\tilde{p}: \tilde{X} \rightarrow \tilde{X} / \tilde{\alpha}$ be the singular part of $p$ and let

$$
\gamma: G \times X \times Y \rightarrow X \times Y, \quad \tilde{\gamma}: G \times \tilde{X} \times Y \rightarrow \tilde{X} \times Y
$$

be the actions associated to $\alpha, \beta$ and $\tilde{\alpha}, \beta$, respectively. Let $f: \tilde{X} \rightarrow Y$ be an equivariant map and $\varphi^{f}: \tilde{X} / \tilde{\alpha} \rightarrow(\tilde{X} \times Y) / \tilde{\gamma}$ be the cross-section associated to $f$. Then $f$ has an equivariant extension $g: X \rightarrow Y$ over $X$ if and only if the cross-section $\varphi^{f}$ has an extension to a cross-section of the fibration $q$.

4. Extending cross-sections in singular fibrations. In view of (3.1), Theorem (1.1) reduces to the following theorem:

(4.1) THEOREM. Let $\alpha$ be an action of a compact Lie group $G$ on a finite dimensional compact metric space with a finite number of conjugacy classes of isotropy subgroups. Let $p=p^{\alpha}: X \rightarrow X / \alpha$ be the corresponding singular fibration and $\tilde{p}: \tilde{X} \rightarrow \tilde{X} / \tilde{\alpha}$ be a closed singular part of $p$. Let $\beta$ be a G-action 
on a compact metric space $Y$ and let $q$ and $\tilde{q}$ be the singular fibrations associated to $p, \beta$ and $\tilde{p}, \beta$ respectively.

(i) If $Y$ is an $A N R$, then any cross-section of $\tilde{q}$ can be extended to a cross-section of $q$ over a neighborhood of $\tilde{X} / \tilde{\alpha}$;

(ii) If $Y$ is an $A R$, then any cross-section of $\tilde{q}$ can be extended to a crosssection of $q$.

OUTLINE OF THE PROOF. Applying Mostow's linearization theorem [5] we can assume that $X$ is equivariantly embedded in a Euclidean space $R^{n}$ with an orthogonal $G$-action.

Let $\overline{R^{n}}$ be the maximal part of $R^{n}$ on which the action is free. Let $E=\overline{R^{n}} \cup X, \bar{E}=E-\tilde{X}, \widetilde{E}=\tilde{X}$. We replace the action $\alpha$ by this orthogonal action on $E$ and continue denoting it by $\alpha$ with the partition $\alpha=(\bar{\alpha} \mid \tilde{\alpha})$ into the regular and singular parts, $\bar{E}$ and $\tilde{E}$, respectively. We also keep the notation $p=(\bar{p} \mid \tilde{p})$ for the corresponding orbit maps. The regular part $\bar{E}$ is an open equivariant subset of $R^{n}$ and the base space $\bar{E} / \bar{\alpha}$ is an open manifold. Let $B=E / \alpha, \bar{B}=\bar{E} / \bar{\alpha}$, and $\widetilde{B}=\widetilde{E} / \tilde{\alpha}$. We would like to replace $\bar{B}$ by an infinite simplicial complex and for this purpose we prove the following lemma (compare Lemma 4.8 of [2]):

(4.2) LEMMA. There exist:

(1) a space $Z$ containing $\widetilde{B}$ as a closed subset;

(2) a finite dimensional locally finite triangulation $K$ of $Z-\widetilde{B}$;

(3) inaps of pairs

$$
\kappa:(B, \bar{B}) \rightarrow(Z,|K|) \quad \lambda:(Z,|K|) \rightarrow(B, \bar{B})
$$

each being the identity on $\widetilde{B}$;

(4) $A$ homotopy $\lambda \circ \kappa \simeq 1_{(B, \bar{B})}$ fixing every point on $\widetilde{B}$.

Now for the proof of (4.1) it suffices to construct a cross-section of the induced singular fibration $\lambda^{*} q$ whose regular part is over the infinite polyhedron $|K|$. This can be done by a stepwise extension on the skeletons of $K$. It is interesting, however, that the finite dimensionality of $K$ plays an essential role in the proof, since a special care is needed for simplices approaching the singular part of $\widetilde{B}$.

\section{BIBLIOGRAPHY}

1. A. Heller, On equivariant maps of spaces with operators, Ann. of Math. (2) 55 (1952), 223-231. MR 14, 1110.

2. J. W. Jaworowski, Equivariant extensions of maps, Pacific J. Math. (to appear).

3. - Extensions of maps in spaces with periodic homeomorphisms, Bull. Amer. Math. Soc. 78 (1972), 527-531.

4. - Equivariant retracts for compact Lie group actions, (to appear).

5. G. D. Mostow, Equivariant embeddings in Euclidean space, Ann. of Math. (2) 65 (1957), 432-446. MR 19, 291.

Department of Mathematics, Michigan State University, East Lansing, Michigan 48823

Current address: Department of Mathematics, Indiana University, Bloomington, Indiana 47401 